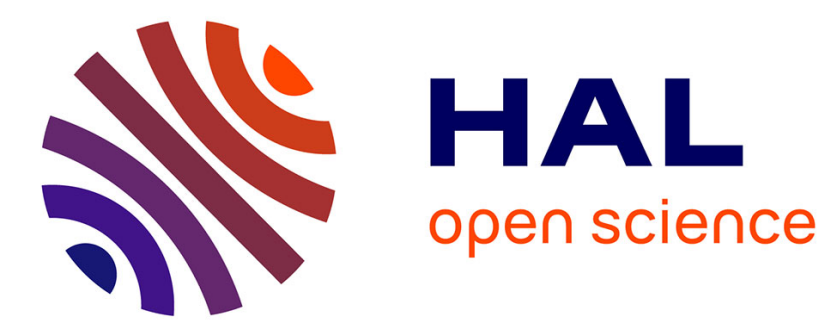

\title{
Mechanical strain mapping of GaAs based VCSELs
}

Merwan Mokhtari, Philippe Pagnod-Rossiaux, Christophe Levallois, Francois

Laruelle, Daniel T Cassidy, Mauro Bettiati, Jean-Pierre Landesman

\section{To cite this version:}

Merwan Mokhtari, Philippe Pagnod-Rossiaux, Christophe Levallois, Francois Laruelle, Daniel T Cassidy, et al.. Mechanical strain mapping of GaAs based VCSELs. Applied Physics Letters, 2021, 118 (9), pp.091102. 10.1063/5.0040386 . hal-03190309

\section{HAL Id: hal-03190309 https://hal.science/hal-03190309}

Submitted on 15 Jun 2021

HAL is a multi-disciplinary open access archive for the deposit and dissemination of scientific research documents, whether they are published or not. The documents may come from teaching and research institutions in France or abroad, or from public or private research centers.
L'archive ouverte pluridisciplinaire HAL, est destinée au dépôt et à la diffusion de documents scientifiques de niveau recherche, publiés ou non, émanant des établissements d'enseignement et de recherche français ou étrangers, des laboratoires publics ou privés. 


\section{Mechanical strain mapping of GaAs based VCSELs}

Merwan Mokhtari, ${ }^{1,}$ a) Philippe Pagnod-Rossiaux, ${ }^{2}$ Christophe Levallois, ${ }^{3}$ François Laruelle, ${ }^{2}$ Daniel T. Cassidy, ${ }^{4}$ Mauro Bettiati, ${ }^{2}$ and Jean-Pierre Landesman ${ }^{3}$

1) Univ Rennes, CNRS, IPR - UMR 6251, F-35000 Rennes, France ${ }^{\mathrm{b})}$

2) $3 S P$ Technologies SAS, 91625 Nozay, France

${ }^{3)}$ Univ Rennes, INSA Rennes, CNRS, Institut FOTON - UMR 6082, F-35000 Rennes, France

4) McMaster University, Department of Engineering Physics, Hamilton, ON L8S 4L7, Canada

(Dated: 2 February 2021)

We report an investigation of the strain field in mesa structures for oxide-confined vertical-cavity surface-emitting lasers (VCSELs) using polarization-resolved micro-photoluminescence $(\mu-\mathrm{PL})$ measurement of the degree of polarization (DOP) at room temperature. The DOP of the PL is correlated to the spatial distribution of the embedded anisotropic strain in a VCSEL structure. Measurements normal to (100) surfaces of the samples and from (110) cross section planes were performed. The effect of two processes required in the fabrication of GaAs based VCSELs were studied: the plasma etching of the P-doped distributed Bragg reflector (DBR); and, the wet oxidation process used to control current flow and lateral optical confinement. The DOP method allows very sensitive measurements of the mechanical strain (in the order of $10^{-5}$ ) accumulated in VCSEL devices even during different steps of the fabrication process.

Remarkable advances in vertical-cavity surface-emitting lasers (VCSELs) have enabled them to support a steadily growing number of applications including high-speed optical interconnects in data centers ${ }^{1}$. In this context, the reliability and performance requirements for these laser diodes are very stringent and have to be taken into account in the epitaxial design, processing, geometry of the laser diode, and operating parameters. Today, both electrical and optical confinement are obtained by selective wet oxidation of $A l_{x} G a_{1-x} A s$ layers within the VCSEL's vertical structure ${ }^{2,3}$. Except for the multi-quantum wells (MQWs), the oxidized layers constitute the main source of concentrated internal mechanical stress in the VCSEL. The induced deformations are critical since they are located close to the active region ${ }^{4,5}$ and their originating dislocations that extend to the active region are identified as major degradation mechanisms ${ }^{6}$. Much work has been done with various strain evaluation techniques for buried oxide structures $^{7-11}$ but analyses were performed on dedicated samples and not on epitaxial growth-complete VCSEL structures.

Measurements of the degree of polarization (DOP) of the micro-photoluminescence ( $\mu$-PL) have been made on epitaxial growth-complete VCSEL structures. These DOP analyses have demonstrated that this technique is effective for strain mapping in VCSELs. These first measurements provided a method which allowed to evaluate with high sensitivity the main components of strain induced by the related critical steps of the VCSEL structure fabrication process. This technique was used to measure the strain field caused by the plasma etching and oxidation in GaAs based VCSEL structures and these findings are reported here.

The samples for this study are $850 \mathrm{~nm}$ VCSEL structures grown by metalorganic vapor phase epitaxy on (100) $2^{\circ}$ off toward $<110\rangle$ GaAs substrates. The Si-doped lower 30-pair

\footnotetext{
a) Present address: 3SP Technologies SAS, 91625 Nozay, France

${ }^{b)}$ Electronic mail: mmokhtari@3spgroup.com
}

distributed Bragg reflector (DBR) and the C-doped upper 16-pair DBR are both made of $A l_{0.12} G a_{0.88} A s / A l_{0.9} G a_{0.1} A s$ pairs. The active region is undoped and consists of $I n_{0.08} G a_{0.92} A s / A l_{0.4} G a_{0.6} A s$ MQWs to obtain emission at 850 $\mathrm{nm}$. On top of the active area, two $A l_{0.98} G a_{0.02} A s$ thin layers $(30 \mathrm{~nm})$ are devoted to the oxidation process to ensure electrical and optical confinement. Following the epitaxial growth, the samples were etched by $\mathrm{SiCl}_{4} / \mathrm{He}$ inductively coupled plasma reactive ion etching in order to form circular or rectangular shaped mesas and expose the $A l_{0.98} G a_{0.02} A s$ layers to the oxidizing species of the next step in fabrication of functioning VCSELs. For the circular geometry the diameters were $25 \mu \mathrm{m}$ and for rectangular geometry the dimensions were $50 \mu \mathrm{m} \times 1000 \mu \mathrm{m}$. The lateral extension of the $\mathrm{Al}_{0.98} \mathrm{Ga}_{0.02} \mathrm{As}$ oxidation was controlled by in-situ optical monitoring to reach $9 \mu \mathrm{m}$ depth. DOP-PL characterization was performed at room temperature using a 635-nm laser for excitation. The excitation spot size was about $2 \mu \mathrm{m}$ and the estimated power density with the use of a 20x magnification objective was close to 1 $\mathrm{kW} / \mathrm{cm}^{2}$. Theory and technical details on this technique can be found in ${ }^{12-15}$.

The DOP signal measured along direction $y$ can be written as:

$$
D O P_{y}=\frac{L_{x}-L_{z}}{L_{x}+L_{z}}=-C_{\varepsilon} \times\left(\varepsilon_{x x}-\varepsilon_{z z}\right)
$$

where $x$ and $z$ denote the two directions perpendicular to $y$, and therefore belong to the plane of the surface being measured. $L_{x}$ and $L_{z}$ are the components of the spectrally integrated PL with polarization along these two directions. $\varepsilon_{x x}$ and $\varepsilon_{z z}$ are the normal crystal deformations. $C_{\varepsilon}$ is a positive calibration constant which can be determined experimentally. For GaAs, the value is known and demonstrated that the sensitivity of the DOP is extremely high with measured deformations in the order of $10^{-5} 13$. For $A l_{0.12} G a_{0.88} A s$, an approximation was made to use the DOP calibration constant of GaAs since $C_{\varepsilon}$ is expected it to scale linearly with the valence band deformation potentials. Based on literature, these values do not change much (less than 3\%) between GaAs and $A l_{0.12} G a_{0.88} A s^{16}$. 
However, DOP is sensitive to non-biaxial deformations perpendicular to the measurement direction, so one can measure only anisotropic strain inside the structure. Typically, for GaAs, a DOP of $1 \%$ corresponds to an anisotropic strain value of $2 \times 10^{-4}$ which corresponds to an anisotropic stress value of $13 \mathrm{MPa}$ if we consider the linear elastic regime.

First, DOP measurements from the (100) surface after plasma etching definition of $25 \mu \mathrm{m}$ circular mesas were performed. Fig. 1 presents the top view PL and DOP intensity maps from the P-DBR of 16 pairs of height and the exposed N-DBR surface at the bottom of the mesa. In this configura-
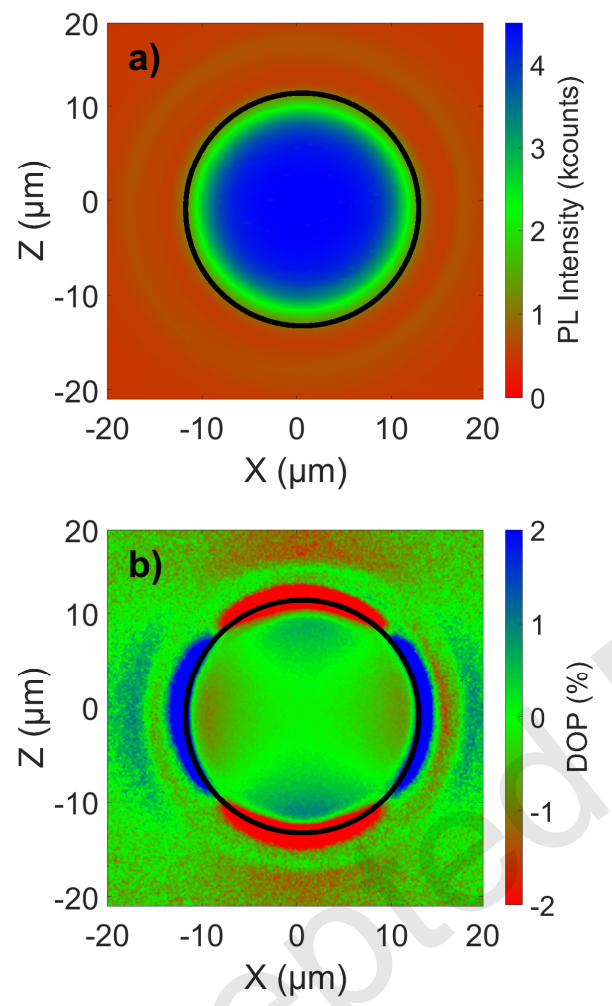

FIG. 1. Maps of the PL (a) and DOP (b) signal of the (100)-surface of a $25-\mu \mathrm{m}$ diameter circular mesa VCSEL structure. Black circles represent the mesa.

tion, the PL signal collected on top of the mesa is mainly coming from the $A l_{0.12} G a_{0.88} A s$ layers of the P-DBR ${ }^{12}$. By symmetry, the presence of interfaces parallel to the (100) surface does not impact the DOP. The DOP signal is very pronounced at the edges of the mesa and decreases towards the mesa center. In this case, as the pattern has rotational symmetry, we do not probe anisotropic deformation at the center of the circular mesa. Also, the induced deformation field in the areas beyond the foot of the etched mesa is detectable even if the signal is noisy. Due to the circular geometry, we can assume that the deformations are essentially caused by the radial displacements at any point. From this assumption, we consider a polar coordinate system with the azimuthal component of the displacement field $u_{\theta}$ equal to zero. On the other hand, due to symmetry reasons, the radial component of displacement $u_{r}$ is a function of only the radius of the considered point. With these assumptions, we have a single scalar function $\left(u_{r}\right)$ depending on only one radius $(r)$. Thus, the formula linking the DOP with the strain tensor components in the cartesian coordinate system can be redefined as a function of the strain tensor components in a cylindrical coordinate system:

$$
D O P=-C_{\varepsilon} \times\left(\varepsilon_{x x}-\varepsilon_{z z}\right)=-C_{\varepsilon} \times \cos (2 \theta) \times\left(\varepsilon_{r r}-\varepsilon_{\theta \theta}\right)
$$

where $\varepsilon_{r r}$ and $\varepsilon_{\theta \theta}$ are the normal components of the strain tensor along the radial direction $r$ and the tangential direction $\theta$ respectively and equal to: $\varepsilon_{r r}=\frac{d u_{r}}{d r} ; \varepsilon_{\theta \theta}=\frac{u_{r}}{r}$. Fig. 2 shows the DOP map obtained by dividing each experimental value of DOP by $\cos (2 \theta)$. These results clearly confirm the radial

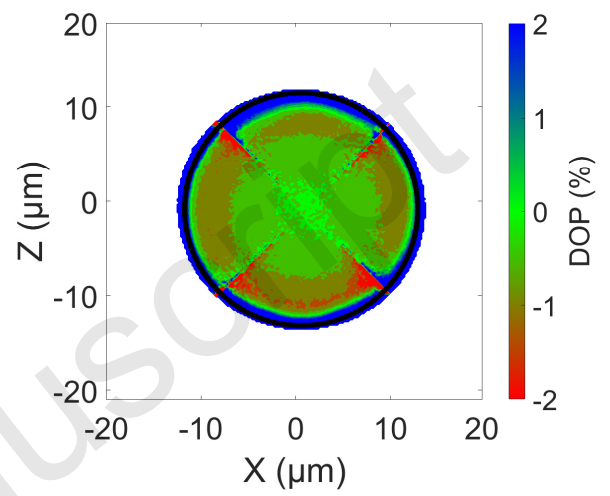

FIG. 2. Map of the DOP signal divided by $\cos (2 \theta)$ of the (100)surface of a $25-\mu \mathrm{m}$ diameter circular mesa VCSEL structure. Black circle represent the mesa. The lines are mathematical artefacts representing the indeterminate areas where the cosine function equals zero.

distribution of the deformation induced by the etching of the circular mesa. Also, for a VCSEL structure, the P-DBR layers are slightly strained in the growth plane (e.g., if we consider the lattice mismatch between $A l_{0.12} G a_{0.88} A s$ and GaAs substrate). Etching the material modifies this initial strain state to the same isotropic symmetry as the symmetry of the mesa.

Then, measurements perpendicular to the (110) facet of a rectangular VCSEL mesa cleaved along the direction transverse to the long segment were performed. Fig. 3 shows the PL and DOP maps measured at the (110) plane cross section of a $50 \mu \mathrm{m}$ wide rectangular mesa. Based on the PL and DOP signals, one can easily distinguish the different areas of the VCSEL structure. The very low DOP values near the top of the mesa (less than $2 \%$ ) are physical artefacts owing likely to the collection, from near the edge of the sample, of light that has undergone polarization-dependent internal reflections. Inside the structure, the measured DOP values are very high, reaching several tens of percent especially near the active area. It is known that in this configuration, as the structure is composed of thin layers, the polarization of the PL will be affected by confinement of carriers from the thin luminescent layers ( $A l_{0.12} G a_{0.88} A s$ in the P- and N-DBR and $I n_{0.08} G a_{0.92} A s$ QWs within the active zone). These layers have then an intrinsic DOP with external strain adding to this intrinsic DOP. As a consequence, mainly the degree of polarization intrinsic to the confinement of carriers in the luminescent layers is visible in 

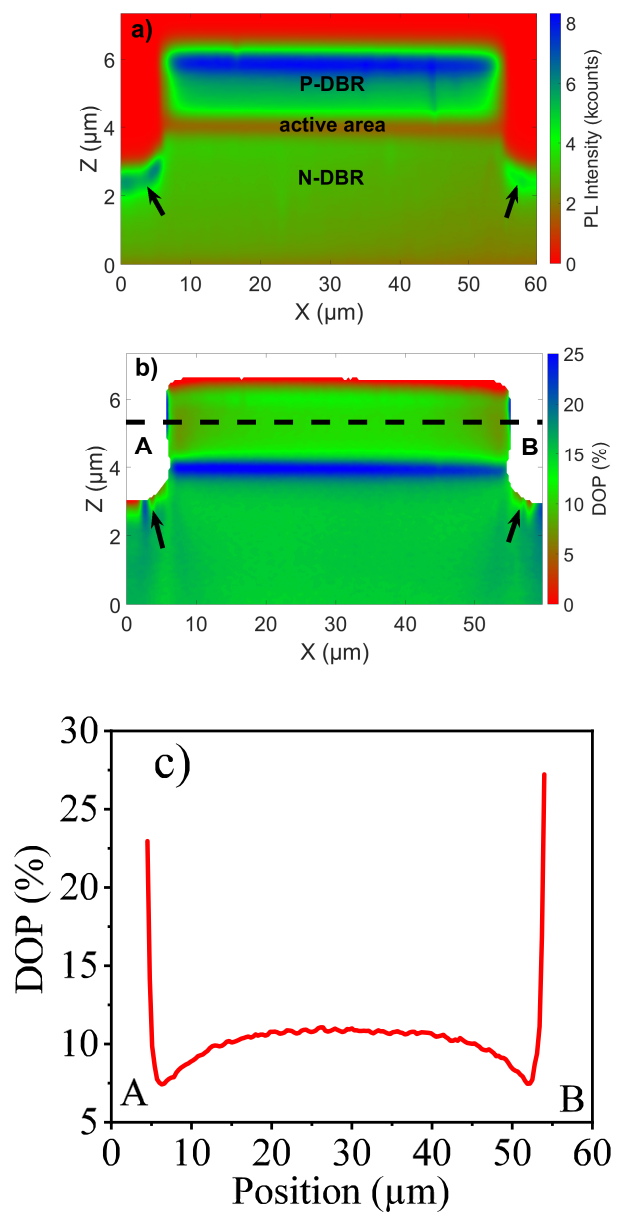

FIG. 3. Maps of the PL (a) and DOP (b) signals of the cleaved (110) facet of the $50 \mu \mathrm{m}$ wide rectangular mesa VCSEL structure. The black arrows indicate the high stressed regions near the mesa foot. (c) Represents the DOP cross-section profile along A-B within the $\mathrm{P}-\mathrm{DBR}$ as indicated in (b).

the false colour image. Indeed, quantum confinement affects the distribution of electrons which leads to the creation of this degree of intrinsic polarization ${ }^{17}$ and explains the important values near the active region.

High values of DOP, indicated by the arrows in Fig. 3 (b), can be observed and associated to an increase of the PL intensity at the foot of the mesa, reflecting the presence of strong concentrated stress that also propagates deep into the bottom N-DBR. Near the etched mesa walls, a variation of the DOP is present which decreases towards the center of the mesa. This behaviour is illustrated by Fig. 3 (c) representing the crosssection profile of DOP from point A to B as indicated in Fig. 3 (b). This change of the DOP signal corresponds to the relaxation of the internal strain in the mesa and therefore is in agreement to the observations of the (100) surface. This qualitative study from the cross-section confirms the effects observed within the mesa measured from the surface after etching: dry etching modifies the initial strain distribution in the VCSEL layers.

Next, DOP measurements on circular $25 \mu \mathrm{m}$ diameter mesas after oxidation were achieved. Fig. 4 presents the top view PL and DOP maps for the mesa. From the PL map, Fig.
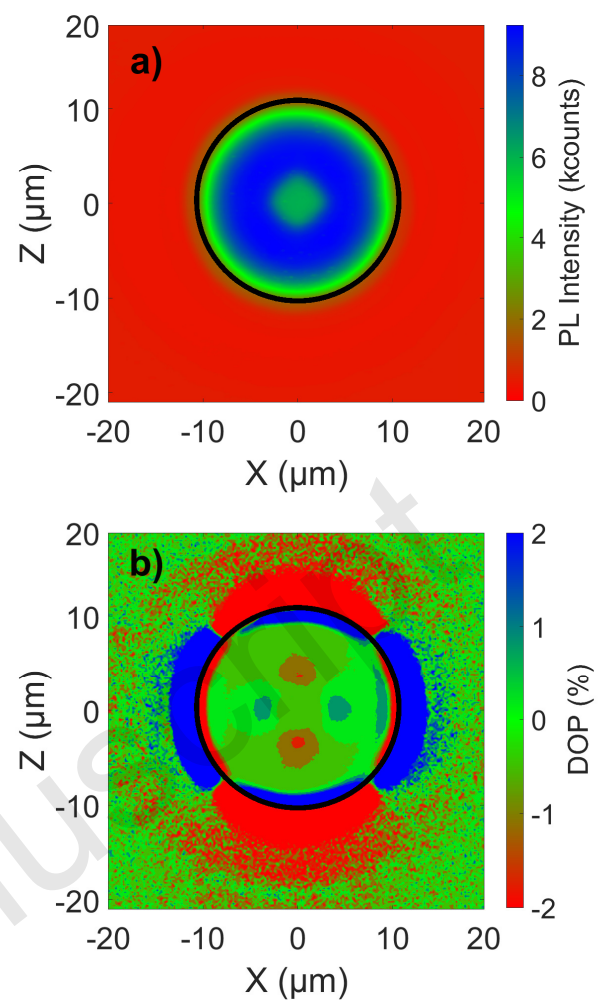

FIG. 4. Maps of the PL signal (a) and DOP (b) signal of the (100) surface of $25-\mu \mathrm{m}$ circular mesa VCSEL structure after oxidation. Black circles represent the mesa.

4 (a), the oxidized and unoxidized areas can easily be distinguished and the oxidation anisotropy effect of the squareshaped aperture at the mesa center is observed. From the DOP map in Fig. 4 (b), we note the same radial distribution of the strain measured before oxidation at the mesa edges. Additionally, high positive and negative DOP values are observed just inside the mesa close to the edges. The accumulated strain present in these regions is a signature of all the oxidized $A l_{0.9} G a_{0.1} A s$ layers in the P-DBR with an oxide depth of about $1.5 \mu \mathrm{m}$. Furthermore, close to the center of the mesa, we observe an increased localized DOP signal from buried $A l_{0.98} G a_{0.02} A s$ oxidized layers fronts and the corners of the square-shaped aperture. These variations are induced by the lower volume density of these buried oxidized layers ${ }^{18}$. The distribution of the strain is therefore no longer isotropic in this case. Based on this observation, we can assume that the shape of the non-oxidized aperture has an influence on the strain distribution in the structure.

Fig. 5 shows the PL and DOP maps measured from the (110) surface of a $50 \mu \mathrm{m}$ wide rectangular mesa after oxidation. The PL signal increases obviously in a certain inner area close to the mesa side wall, at this depth, the only difference to the center of the structure is the presence of the oxide. It is clear that the intensity of the PL from the luminescent $A l_{0.12} G a_{0.88} A s$ layers and the MQWs is influenced by the 

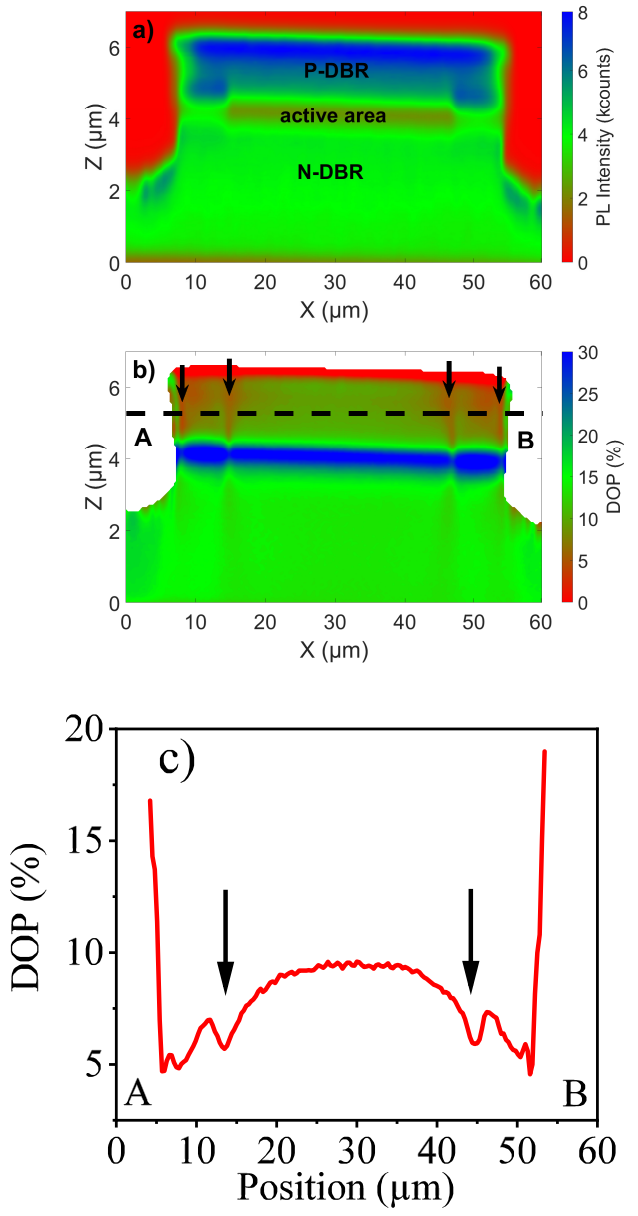

FIG. 5. Maps of the PL (a) and DOP (b) signals from the (110) surface of the $50 \mu \mathrm{m}$ rectangular mesa VCSEL structure. The solid black arrows indicate the horizontal position of the buried $A l_{0.98} G a_{0.02} A s$ oxidized layers fronts. The dot black arrows indicate the horizontal position of the $A l_{0.9} G a_{0.1} A s$ P-DBR oxidized layers fronts. (c) Represents the DOP cross-section profile along A-B within the P-DBR as indicated in (b).

presence of the oxidized layers. From the DOP map, one can see some additional variation near the side walls of the mesa in the P-DBR. This DOP signal variation is correlated to the strain generated by the oxidation of the P-DBR $A l_{0.9} G a_{0.1} A s$ layers and verifies the effect observed from the (100)-surface. Moreover, in the region of the buried $A l_{0.98} G a_{0.02} A s$ oxidized layers and close to the MQWs, we can clearly see some DOP variation related to the strain induced by the oxidation. As a consequence, we observe the associated strain field emerging from the oxidation fronts and propagating along both $\mathrm{P}$ and N-DBR. This effect is better illustrated in Fig. 5 (c) which represents the cross-section DOP profile along the mesa (section along $\mathrm{AB}$ ) in the $\mathrm{P}-\mathrm{DBR}$, showing two peaks which correspond to the oxidation fronts from both sides. This observation confirms the fact that we were able to probe the induced effect of the mechanical strain concentrated at the level of the buried oxidized layers far from the (100) surface (about $2 \mu \mathrm{m}$ below the surface). This qualitative study from the cross- section supports the observations made from the surface after oxidation: the oxidation process creates strain at the mesa edges and at the oxidation fronts deep inside the mesa. The magnitudes of these strains are technologically important and are easily characterized by measurement of the DOP of PL from either the (100) growth surface or a (110) cleaved facet.

Using the polarization-resolved $\mu$-PL technique on a VCSEL structure after plasma etching and after oxidation allowed us to map with high sensitivity the strain induced by key processing steps. By coupling the DOP non-destructive measurements of the (100) surface with measurements of the (110) facet, we are able to obtain an overall and precise measurement of the strain spatial distribution within an oxidized VCSEL structure.

We could see with this technique that each step induces mechanical strain in different areas of the VCSEL structure. The plasma etching of the P-DBR removes material which requires the strain within the mesa to relax at the free surface. The wet thermal oxidation step induces two different effects. The first one is the large strain accumulated at the edges of the mesa caused by the oxidation of the $A l_{0.9} G a_{0.1} A$ s layers present in the P-DBR. The second one is the strain generated by the buried oxidized layers. The associated deformation field emerges from the oxidation front and propagates throughout the VCSEL structure. Anisotropic strain values up to $1.2 \times 10^{-3}$ corresponding to an anisotropic stress value of $80 \mathrm{MPa}$ were measured near the oxidation fronts of the $A l_{0.98} G a_{0.02} A s$ buried oxidized layers. For reliability concerns, this can be critical for the device since it is located close to the core of the laser. More generally, strain variations were measured from the surface, $2 \mu \mathrm{m}$ away of the root cause demonstrating that the DOP technique can be used for in-situ mechanical strain monitoring during fabrication of semiconductor lasers devices.

\section{DATA AVAILABILITY}

The data that support the findings of this study are available from the corresponding author upon reasonable request.

\section{REFERENCES}

${ }^{1}$ A. Liu, P. Wolf, J. A. Lott, and D. Bimberg, Photon. Res. 7, 121 (2019).

${ }^{2}$ D. L. Huffaker, D. G. Deppe, K. Kumar, and T. J. Rogers, Appl. Phys. Lett. 65, 97 (1994).

${ }^{3}$ K. Choquette, K. Geib, C. Ashby, R. Twesten, O. Blum, H. Hou, D. Follstaedt, B. Hammons, D. Mathes, and R. Hull, IEEE J. Select. Topics Quantum Electron. 3, 916 (1997).

${ }^{4}$ K. D. Choquette, K. M. Geib, H. C. Chui, B. E. Hammons, H. Q. Hou, T. J. Drummond, and R. Hull, Appl. Phys. Lett. 69, 1385 (1996).

${ }^{5}$ C. J. Helms, I. Aeby, W. Luo, R. W. Herrick, and A. Yuen, in Vertical-Cavity Surface-Emitting Lasers VIII, Vol. 5364, edited by C. Lei, K. D. Choquette, and S. P. Kilcoyne (SPIE, Bellingham, WA, San Jose, CA, United States, 2004) p. 183.

${ }^{6}$ R. W. Herrick, A. Dafinca, P. Farthouat, A. A. Grillo, S. J. McMahon, and A. R. Weidberg, IEEE J. Quantum Electron. 49, 1045 (2013).

${ }^{7}$ R. Keller, A. Roshko, R. Geiss, K. Bertness, and T. Quinn, Micro. Engn. 75, 96 (2004). 
${ }^{8}$ F. Chouchane, G. Almuneau, O. Gauthier-Lafaye, A. Monmayrant, A. Arnoult, G. Lacoste, and C. Fontaine, Appl. Phys. Lett. 98, 261921 (2011).

${ }^{9}$ J. P. Landesman, A. Fiore, J. Nagle, V. Berger, E. Rosencher, and P. Puech, Appl. Phys. Lett. 71, 2520 (1997).

${ }^{10}$ A. C. Alonzo, X.-C. Cheng, and T. C. McGill, Journal of Applied Physics 87, 4594 (2000)

${ }^{11}$ A. Strittmatter, A. Schliwa, J.-H. Schulze, T. D. Germann, A. Dreismann, O. Hitzemann, E. Stock, I. A. Ostapenko, S. Rodt, W. Unrau, U. W. Pohl, A. Hoffmann, D. Bimberg, and V. Haisler, Appl. Phys. Lett. 100, 093111 (2012).

${ }^{12}$ M. Mokhtari, P. Pagnod-Rossiaux, F. Laruelle, J.-P. Landesman, A. Moreac, C. Levallois, and D. T. Cassidy, J. Electron. Mater. 47, 4987 (2018).
${ }^{13}$ D. Lisak, D. Cassidy, and A. Moore, IEEE Trans. Comp. Packag. Technol. 24, 92 (2001).

${ }^{14}$ D. T. Cassidy, S. K. K. Lam, B. Lakshmi, and D. M. Bruce, Appl. Opt. 43, 1811 (2004)

${ }^{15}$ M. Mokhtari, Ph.D. thesis, University Rennes-1, Rennes (2019).

${ }^{16} \mathrm{~S}$. Adachi, GaAs and Related Materials: Bulk Semiconducting and Superlattice Properties (WORLD SCIENTIFIC, Singapore, 1994).

${ }^{17}$ B. Lakshmi, D. T. Cassidy, and B. J. Robinson, J. Appl. Phys. 79, 7640 (1996).

${ }^{18}$ O. Durand, F. Wyckzisk, J. Olivier, M. Magis, P. Galtier, A. De Rossi, M. Calligaro, V. Ortiz, V. Berger, G. Leo, and G. Assanto, Appl. Phys. Lett. 83, 2554 (2003). 